\title{
PHASE INVERSION AND VISCOSITY IN PIPE FLOW
}

\section{Laura Edwards ${ }^{1}$, Dhurjati Prasad Chakrabarti ${ }^{2 *}$}

\author{
${ }^{1,2}$ Department of Chemical Engineering, The University of The West Indies, St Augustine, \\ Trinidad and Tobago. \\ 1Email: laur_edwards@hotmail.com \\ ${ }^{2}$ Email: dhurjati.chakrabarti@sta.uwi.edu*(Corresponding author)
}

\begin{abstract}
This project is primarily aimed at determining the phase inversion of oil water dispersion from a three-phase mixture comprising of an oil-water emulsion at different oil percentages and air at varying liquid and gas flowrates through a horizontal pipe. Parameters affecting phase inversion were investigated such as liquid-side pressure drop, viscosity and flow regimes. The liquid-side pressure drop was estimated and predicted from existing as well as developed correlations. The results obtained were tabulated and represented graphically. Viscosity measurements were taken at the different oil percentage values. At inversion, the mixture's continuous phase switched from water to oil as indicated by a vast increase in viscosity. A significant increase in pressure drop was noted for all correlations used beyond the point of phase inversion. It was also found that the occurrence of phase inversion affected the flow regimes of the mixture causing a shift from a plug dominant flow to a more slug-type flow pattern.
\end{abstract}

Key words: three phase flow, emulsion, horizontal pipe, phase inversion, oil-water.

https://doi.org/10.47412/RORH7651

\section{Introduction}

Multiphase flow is the general term used to define the simultaneous flow of two or more phases. It is being encountered in many industries. Therefore, the knowledge of the hydrodynamics of flow is significant. Emulsions or the dispersion of one immiscible phase into other, are found in oil production and processing facilities where crude oil can be found to be mixed with water in the presence of naturally occurring emulsifiers. Oil is always be produced together with water from the reservoir and towards the end of the reservoir life, the amount of produced water will increase, especially if the reservoir is driven by natural water aquifer. This scenario will induce the formation of water-in-oil emulsions. If water increases and act as a continuous phase, then it would be oil-in-water dispersion. The change of continuous phase from oil to water or water to oil is termed as phase inversion [1].However, the majority of the studies are confined to batch processes and in flow systems, the studies have been influenced by the information obtained from stirred vessels [2-4]. Most of the equations to predict inversion are empirical in nature [5,6] and the only analysis by Brauner and Ullmann [7] has attempted to predict the inversion point from the principle of minimization of system free energy, combined with a model to predict drop size in dense dispersions. Chesters and Issa [8] have presented a mathematical model to describe the evolution of phase inversion processes in two-fluid systems. The model has been developed within the framework of the two-fluid model for dispersed flows, using Computational Fluid Dynamics (CFD) techniques. The experimental identification of inversion has primarily been carried out through visual observations [9-11], measurement of effective conductivity of the mixture $[12,13]$ and estimation of pressure gradient [14]. However no conclusive results could be obtained primarily due to the difficulty of flow visualization at the high phase velocities. The study by Hapanowicz and Troniewski [15] have reported that the pressure gradient in the water dispersed in oil flow pattern exhibits significant deviations from the predictions of the homogeneous 
flow model and have suggested suitable viscosity corrections to improve the predictions. They have also attempted to incorporate the influence of flow pattern in the analysis of holdup and pressure drop during liquid-liquid flow. Pal [16] suggested that the decrease in pressure gradient of the dispersed flow as compared to the single phase values may be due to the drag reduction phenomenon where the drag reduction is higher in case of oil continuous flows [2]. He attributed this phenomenon to the effect of droplet breakup and coalescence on turbulence in unstable dispersions. It is needless to say that further investigations are required to develop a clear understanding of the phenomenon of phase inversion.

\section{Experimental Procedure}

A schematic representation of the experimental flow loop is shown in Fig 1. Two taps of the manometer with $\mathrm{CCl}_{4}$ as manometer-fluid are attached to the pipe and placed $1.5 \mathrm{~m}$ apart. Thus pressure reading is generated by the bottom liquid phase. The stirrer/mixer is kicked off to begin the mixing of oil (at $32^{\circ} \mathrm{C}$ and $1 \mathrm{~atm}$. pressure, $\rho=907.78 \mathrm{~kg} / \mathrm{m}^{3}, \mu=52 \mathrm{cP}$, surface tension $=31$ dynes $/ \mathrm{cm}$ ) and water in the tank. The oil is chosen as soybean oil to avoid any environmental hazard of petroleum oil. Simultaneously high viscosity like crude oil is observed during flow. The entire pipe section $(5 \mathrm{~m})$ with $50 \mathrm{~mm}$ internal diameter, is made of PVC except for the transparent visual section $(1 \mathrm{~m})$. The control valve, V1, after the pump and the bypass recycle valve, V2, are adjusted to control the flow through the rotameter. The pumped liquid is allowed to circulate and make its way back into the tank. The liquid is allowed to pass through a rotameter and the gas is circulated from the compressor via a rotameter. Both the rotameters are calibrated prior to experiment. The system is allowed to reach a steady state before initiate any reading.

Flow patterns are observed in the transparent tube. Visual observation and Sony HDR-CX240 camera is used to record the flow pattern. The air flow rate is varied between .05 to $0.6 \mathrm{~m} / \mathrm{s}$. The runs are then repeated for liquid flow rate up to $1.25 \mathrm{~m} / \mathrm{s}$. This entire procedure is repeated for various oil percentages ranging from $0-90 \%$. Viscosities of different mixtures are estimated by the Brookfield DV-E viscometer. At these percentages of oil, the continuous phase from water to oil. Fig. 2 describes the cross sectional view of a) air and water continuous phase and $b$ ) air and water dispersed phase.

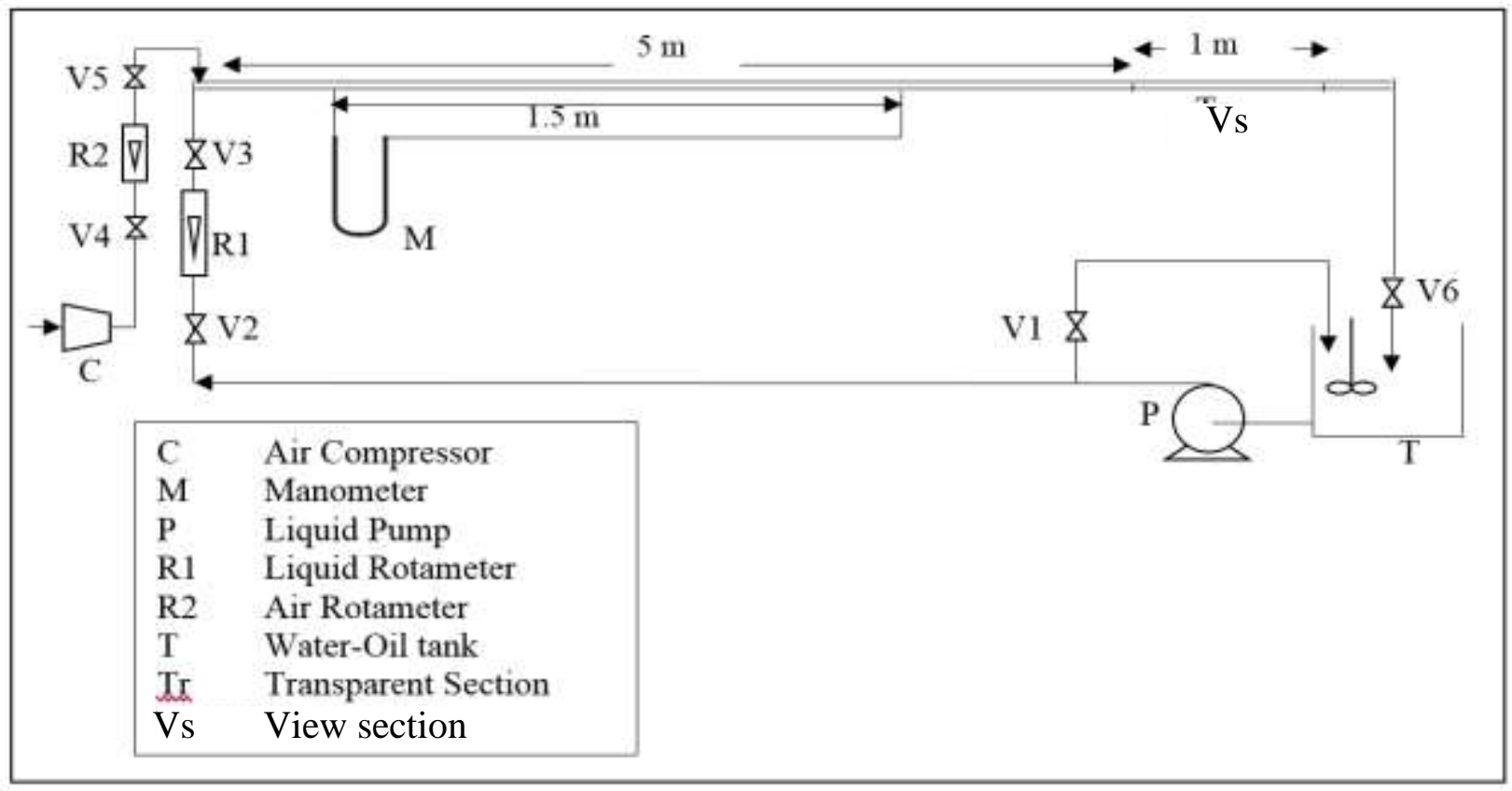

Figure 1: Test rig for multiphase flow 


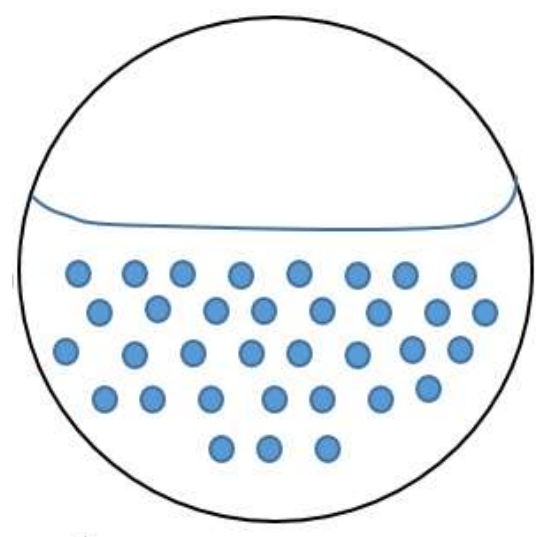

a)

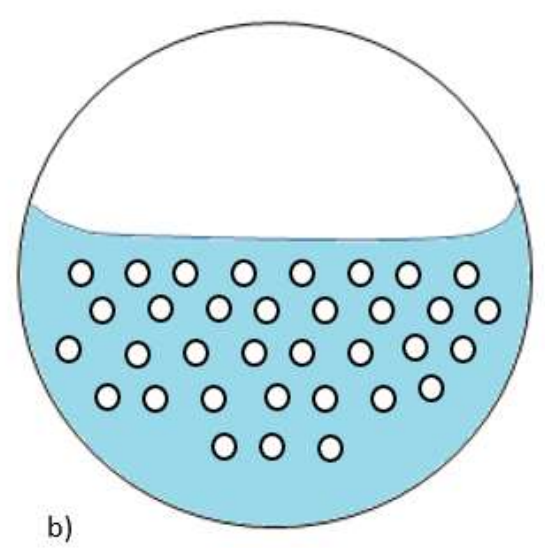

Figure 2: a) water-continuous and b) oil-continuous phase in a pipe

\section{Results and Discussion}

The flow regimes identified for all the variations of oil content at low phase velocities (superficial velocity of air ranging from $0.01-0.11 \mathrm{~m} / \mathrm{s}$ and superficial velocity of liquid mixture ranging from $0.01-0.1 \mathrm{~m} / \mathrm{s}$, given in Fig 3) are stratified smooth (SS), Stratified stratified-wavy small (SSW), stratified wavy (SW), stratified wavy with ripples (SWR) [17]. For higher liquid velocities (superficial velocity of air maintained up to $0.5 \mathrm{~m} / \mathrm{s}$ and superficial velocity of liquid mixture controlled up to $1.23 \mathrm{~m} / \mathrm{s}$, given in Fig 3) flow patterns observed are dispersed slug/elongated bubble (DS/EB) and plug/dispersed gas (PL/DG). The flow regime was classified as DS/EB when the liquid occupied the entire pipe and, intermittently, long air bubbles occupied the top of the pipe. The flow regime was classified as PL/DG when the liquid occupied the entire pipe and, periodically, small air bubbles would occupy the top of the pipe (Fig 3). For higher phase velocities SSW and SWR are not used to designate flow pattern, in lieu of that SW has been used.

In this work no emphasis is given to find the inversion point, however, the work is done to check the effects of phase inversion on flow pattern or pressure drop and hold up. Two different oil cuts are tried for this purpose, $40 \%$, where water is a continuous phase and $65 \%$ where oil is a continuous phase. Fig 4 and 5 represent the flow patterns at this two different cuts. The change in flow pattern may be attributed to the increased effective liquid viscosity upon addition of the oil. This increased viscosity is said to have dampened the flow instability. The applied flow instability, i.e. the air flow, is not enough to allow the shear effects of the air to overcome the gravitational and viscous effects of the liquid. So from $40 \%$ to $65 \%$ oil cut elongated bubble region becomes narrow to give a bigger area of plugs. 


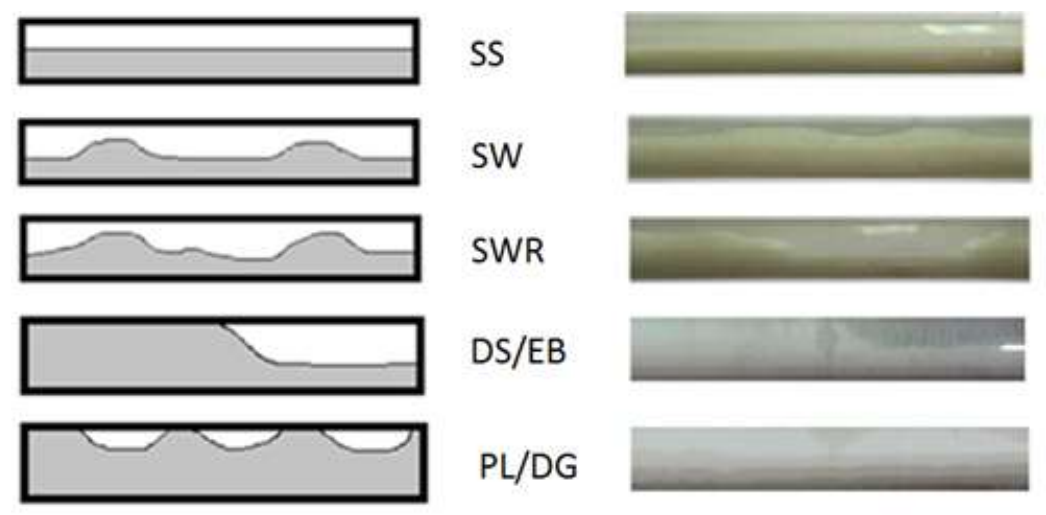

Figure 3: Flow patterns observed

These flow regime phenomena can be attributed to the fact that the oil and water are mixed into an emulsion with the oil being dispersed into the water or vice versa as fine drops that coalesced only at rest. The emulsion/dispersion appeared to be in equilibrium since the oil did not fully separate from the dispersed phase. This might be due to the intensive mixing in the sump that prevents coalescence of the droplets after being fed to the test section. It can be said that the system behaves like a gas - liquid two phase flow, which can justify the use of the weighted average liquid properties. Within the liquid it moves from water continuous to oil continuous phase. However, the variation of viscosity not an expression of additive property [17]. Moreover, the viscosities at different water cut has been applied for completion of momentum balance equation as described (eq. 1-11) by Taitel and Dukler [14]. No better result is obtained by doing so, to justify the change in flow regime with different oil percentage. The change in velocity gradient with respect to viscosity is needed to be looked further analytically. To estimate liquid holdup or pressure drop, Taitel and Dukler [14] theory is extended for calculation.

Referring to Figs. $2 \mathrm{a} \& 2 \mathrm{~b}$, the flow of three fluids, water, oil and gas, is considered. The water is heavier than the oil and occupies the bottom of the pipe. The oil flows in the middle and the gas at the top for stratified flow. A momentum balance for each phase can be written as follows:

$$
\begin{aligned}
& -A_{W}\left(\frac{d p}{d x}\right)-\tau_{w} S_{w}+\tau_{i} S_{i}-\rho_{W} A_{W} g \sin \beta=0 \\
& -A_{O}\left(\frac{d p}{d x}\right)-\tau_{O} S_{O}-\tau_{i} S_{i}+\tau_{j} S_{j}-\rho_{O} A_{O} g \sin \beta=0 \\
& -A_{G}\left(\frac{d p}{d x}\right)-\tau_{G} S_{G}+\tau_{i} S_{i}-\rho_{G} A_{G} g \sin \beta=0
\end{aligned}
$$

In this present case as oil is dispersed into water or vice versa, the dispersed phase is considered as a single liquid phase (Fig. 2). Physical properties of liquid are taken as additive properties of oil and water except viscosity, depending on their fraction. Viscosity is calculated based on Laura et al.,[17]. Momentum balance for the liquid phase will be:

$$
\begin{aligned}
& -A_{L}\left(\frac{d p}{d x}\right)-\tau_{L} S_{L}+\tau_{i} S_{i}-\rho_{L} A_{L} g \sin \beta=0 \\
& \tau_{L}=f_{L}\left(\frac{\rho_{L} U_{L}{ }^{2}}{2}\right) \\
& \tau_{G}=f_{G}\left(\frac{\rho_{G} U_{G}{ }^{2}}{2}\right) \\
& \tau_{i}=f_{i} \frac{\rho_{G}\left(U_{G}-U_{L}\right)^{2}}{2} \\
& f=C R e^{-n}
\end{aligned}
$$

Where $\mathrm{A}$ is a cross sectional area, $\rho$ is density, $\mathrm{P}$ is pressure and $\beta$ is the inclination angle, positive for upward inclination. The subscripts are: $\mathrm{W}$ for water, $\mathrm{O}$ for oil, $\mathrm{G}$ for gas and $\mathrm{L}$ for oil in water emulsion. 
For the present case, three shear stresses are needed to be solved: $\tau_{L}$, the shear stress acting on the wall wetted by the liquid phase $S_{L} ; \tau_{G}$, the shear stress acting on the wall wetted by the gas $S_{G} ; \tau_{i}$, the shear stress acting on the liquid and gas interface $S_{\mathrm{i}}$.

For the shear stresses $(\tau)$ between the liquids or gas and the pipe surface, the friction factors, $f_{L}, f_{W}$ and $f_{i}$ can be approximated by the correlation, where $\mathrm{C}=0.046, \mathrm{n}=0.2$ for turbulent flow, and $\mathrm{C}=16, \mathrm{n}=1$ for laminar flow. The Reynolds number with the concept of hydraulic diameter is defined as

$$
\begin{aligned}
R e_{L} & =4 \frac{A_{L}}{S_{L}} \frac{\rho_{L} U_{L}}{\mu_{L}} \\
R e_{G} & =4 \frac{A_{G}}{\left(S_{G}+S_{i}\right)} \frac{\rho_{G} U_{G}}{\mu_{G}}
\end{aligned}
$$

The expression of liquid viscosity $\left(\mu_{L}\right)$ is

$$
\mu_{L}=2.55 x-0.057 x^{2} \text { in } \mathrm{cP}[17]
$$

Where $x=$ oil $\%$.

For the interfacial gas-liquid shear stress we used a constant value of $f_{i}=0.014$ but if the value off $f_{G}$ is larger than $f i$, then $f_{i}=f_{G}$ is used.

Pressure drop can be eliminated from equations 3 and 4 to yield

$$
-\frac{\tau_{L} S_{L}}{A_{L}}+\frac{\tau_{G} S_{G}}{A_{G}}+\tau_{i} S_{i}\left(\frac{1}{A_{L}}+\frac{1}{A_{G}}\right)-\left(\rho_{L}-\rho_{G}\right) g \sin \beta=0
$$

Equation 12 is solved to get holdup and pressure drop.

In Figs. $4 \& 5$ experimental flow patterns are compared. In the present study oil-water together behaves as a single phase and as a result it resembles a two phase system.

All studies and theories are inferred based on two phases, liquid and gas. However, if the liquid phase is a mixed phase, effect on stratification is not clarified in preceding research. With the change of oil percentage or in other words with the change in viscosity in the liquid phase, different flow regime emerges in the stratified pattern. This can be strongly said as the novel part of the work.

At this range of velocity, the prediction of pressure drop (Fig. 6) and liquid holdup (Fig. 7) are not as perfect as smooth stratified flow for an air-water flow situation $[14,17,18]$. The reason may again be due to flow pattern variations with oil percentage. In some cases, prediction exceeded $+31 \%$ and may be due to the sudden change in viscosity of the liquid phase as well as deviation from ideality. 


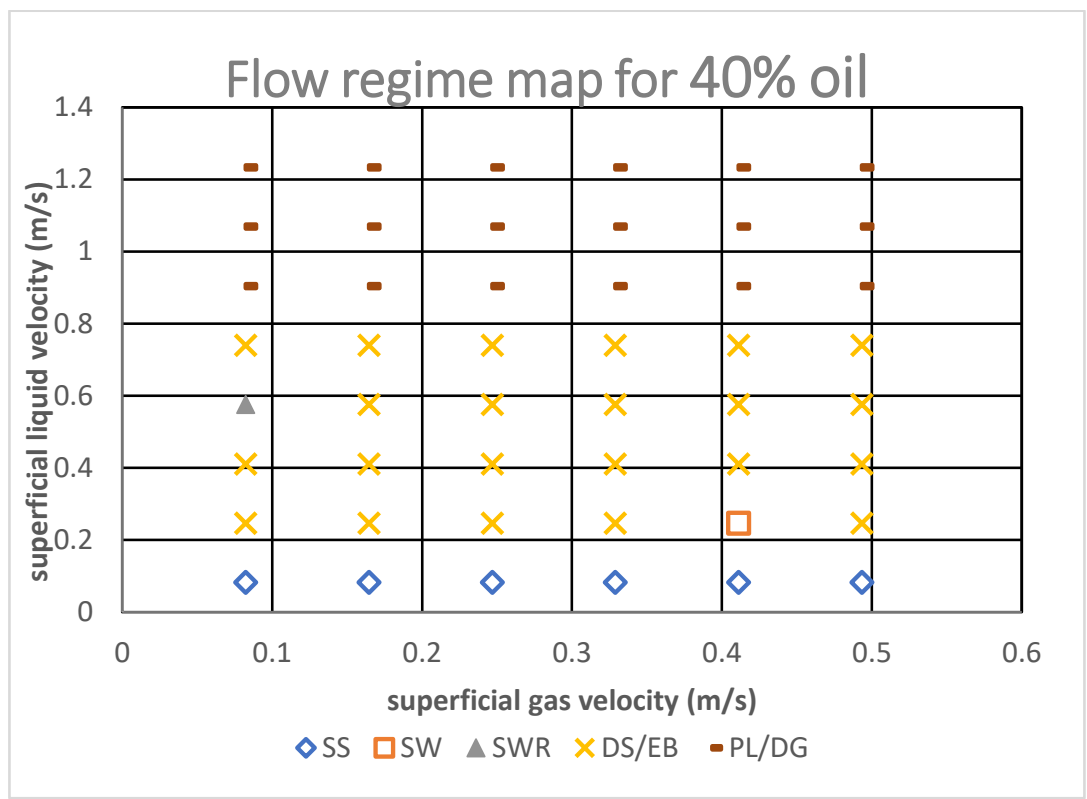

Figure 4: Flow regime map at 40\% oil cut

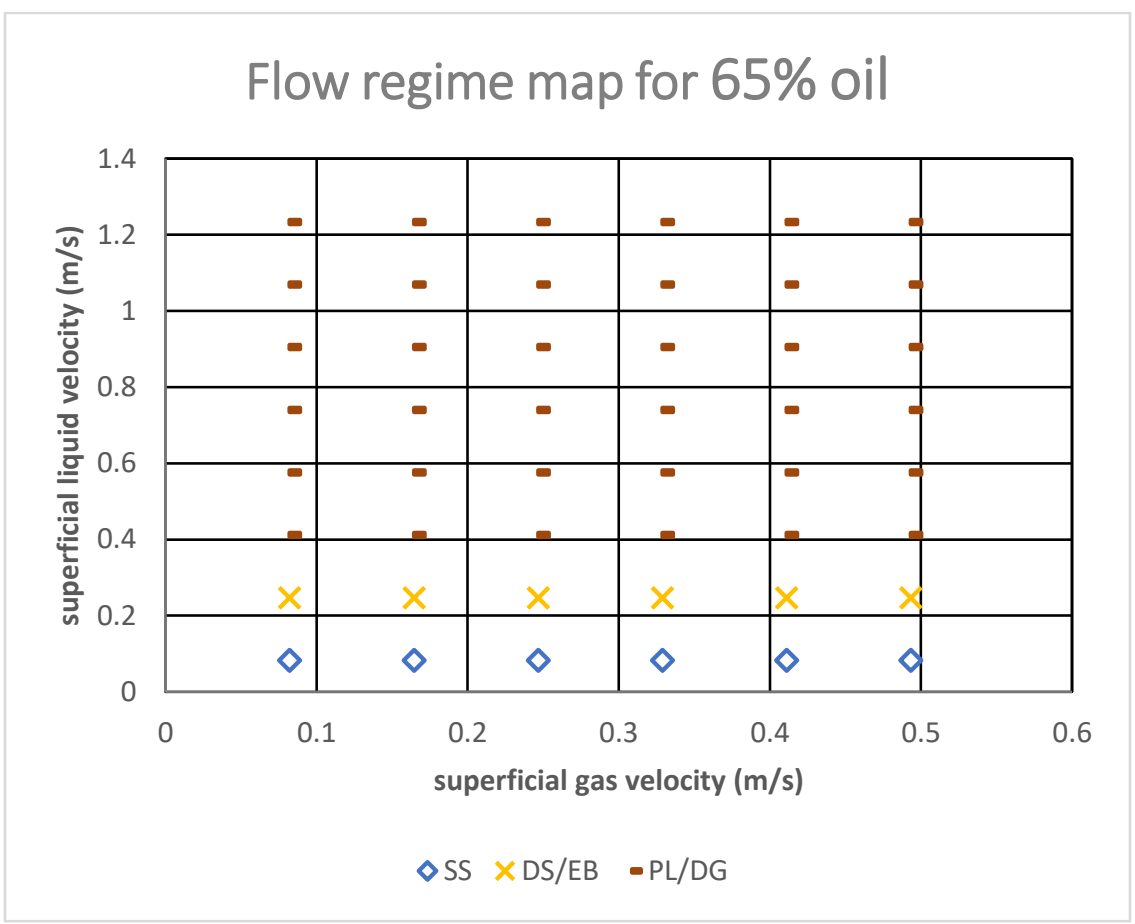

Figure 5: Flow regime map at 65\% oil cut 


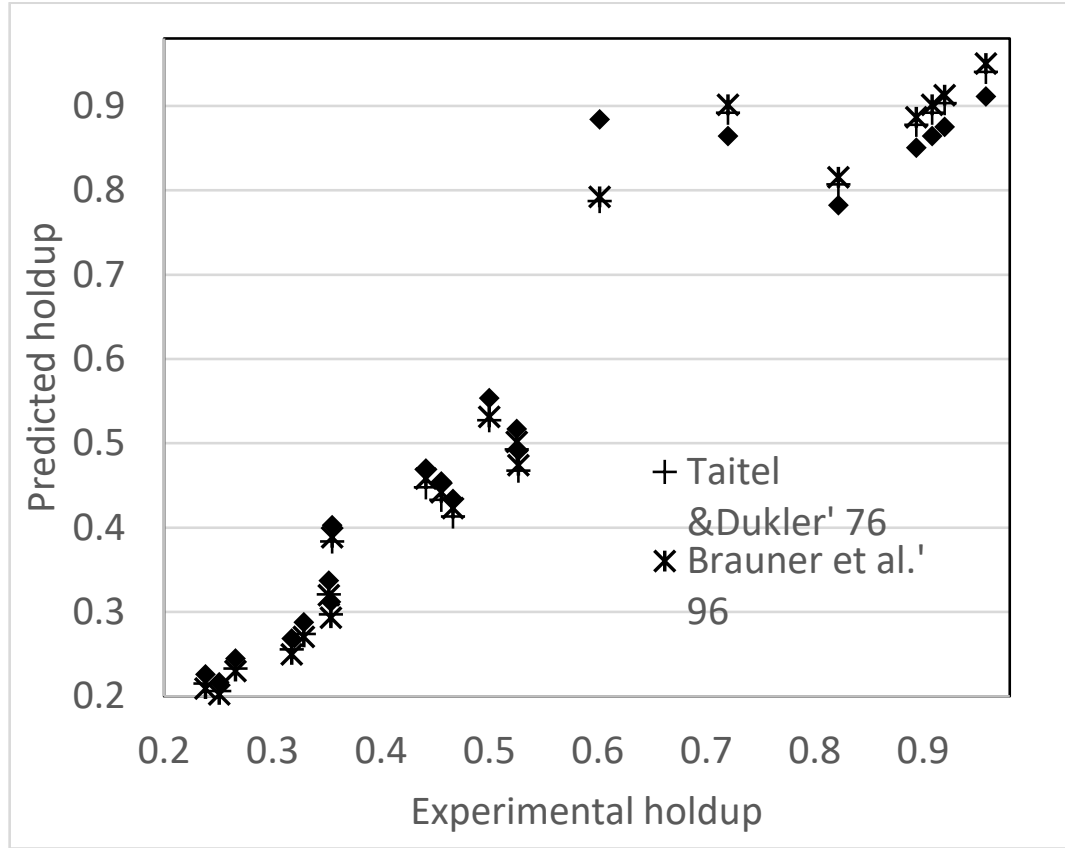

Figure 6: Holdup and prediction

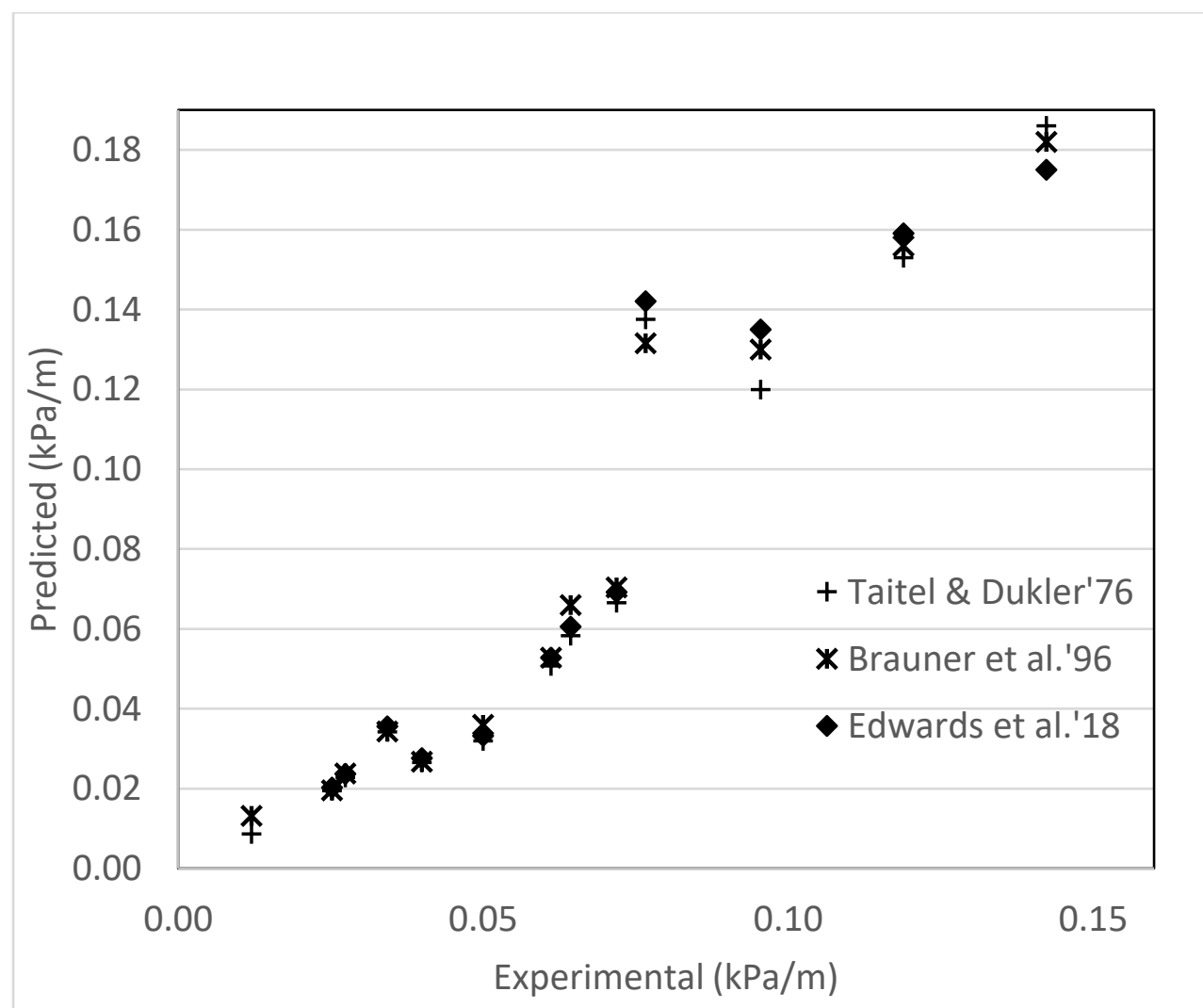

Figure 7: Pressure drop and prediction 


\section{Conclusion and Recommendation}

In the present study, the liquid is a dispersion of oil in water as well as inverted water dispersion. The addition of oil to the liquid phase causes a dampening effect on the flow regime transition, most considerably from stratified to non-stratified flow. In addition to the flow regime, pressure drop and liquid hold up are also compared with previous theory and our predictions of stratification. As the stratification and deviation are not ordered in the present study, experimental data are deviated largely from prediction up to $\pm 31 \%$. This deviation is also resulted from lower or higher flow rates of liquid, not occupying the whole pipe cross section in the absence of air flow. Addition of oil in water increases the region of flow pattern in the flow regime map that is occupied by plugs. Nevertheless, this phenomenon is not following any consistent pattern. The shift is more significant for the inverted phase.This investigation is done mostly by normal video camera and image processing. It is recommended to have high speed camera, conductivity probe or optical probe for flow pattern detection in a finer way.

\section{References}

[1] D.P. Chakrabarti, G. Das, P. K. Das. The Transition from Water Continuous to Oil Continuous Flow Pattern. AIChE J 59 no 11 (2006) 3668-3678.

[2] A. H. Selker, Jr C. A. Sleicher. Factors affecting which phase will disperse when immiscible liquids are stirred together, Canadian Journal of Chemical Engineering 43 no 6 (1965) 298-301.

[3] M. A. Norato, C. Tsouris, L. L. Tavlarides. Phase inversion Studies in Liquid-Liquid Dispersions. Canadian Journal of Chemical Engineering. 76 no 3 (1998) 486-494.

[4] J. M. Zaldivar, M. A. Alos, E. Molga, H. Hernandez, K. R. Westerterp. The effect of phase inversion during semibatch aromatic nitrations. Chemical Engineering and Processing. 34 (1995) 529-542.

[5] S. Arirachakaran, K. D. Oglesby, M. S. Malinowsky, O. Shoham, J. P. Brill. An analysis of oil/water flow phenomena in horizontal pipes. In: SPE Paper 18836, SPE Prod. Operating Symp., Oklahoma, March 13-14, 1989; 155-167.

[6] G. Yeh, F. H. Haynie Jr, R. E. Moses. Phase-volume relationship at the point of phase inversion in liquid dispersions. AIChE Journal. 10 no 2 (1964) 260-265.

[7] N. Brauner, A. Ullmann. Modelling of phase inversion phenomenon in two-phase pipe flow. International Journal of Multiphase Flow 28 no 7, (2002) 1177-1204.

[8] A. K. Chesters, R. Issa. A Framework for the Modelling of Phase Inversion in Liquid-Liquid Systems. 5th International Conference on Multiphase Flow, ICMF’04. Yokohama, Japan, May 30-June 4, 2004. Paper No. 271.

[9] T. W. F. Russell, M. E. Charles. The effect of the less viscous liquid in the laminar flow of two immiscible liquids. Canadian Journal of Chemical Engineering 37 no 1 (1959) 18-24.

[10] D. P. Chakrabarti, G. Das, P. K. Das. Liquid-Liquid Two-Phase Flow through an Orifice; Chemical Engineering \& Technology 196 no 9 (2009) 1117-1129.

[11] D. P. Chakrabarti. Prediction of Phase Split in Horizontal T-Junctions: Revisited; Chemical Engineering \& Technology, 37 no 10 (2014) 1813-1816.

[12] P. Vigneaux, P. Chenais, J. P. Hulin. Liquid-liquid flows in an inclined pipe. AIChE Journal 34 no 5 (1988) 781-789.

[13] Angeli P, Hewitt GF. Flow structure in horizontal oil-water flow. International Journal of Multiphase Flow 26 no 7 (2000) 1117-1140.

[14] Y. Taitel, A. E. Dukler. A model for predicting flow regime transitions in horizontal and near horizontal gas-liquid flow 22 no 1 (1976) 47-55.

[15] J. Hapanowicz, L. Troniewski. Two-phase flow of liquid-liquid mixture in the range of the water droplet pattern. Chemical Engineering and Processing 41 no 2 (2002) 165-172. 
[16] R. Pal, Pipeline flow of unstable and surfactant-stabilized emulsions. AIChE Journal 39 no 11 (1993) $1754-1764$.

[17] L. Edwards, D. Jebourdsingh, D. Dhanpat, D. P. Chakrabarti, Hydrodynamics of air and oil-water dispersion/ emulsion in horizontal pipe flow with low oil percentage at low fluid velocity. 1., Cogent Engineering 5 (2018) 1494494.

[18] N.Brauner, J.Rovinsky, D.Moalem Maron. Determination of the interface curvature in stratified twophase systems by energy considerations. Into J Multiphase Flow 22 no 6 (1996) 1167-1185. 